\title{
Quantitative Discrimination of Effective Porosity Using Digital Image Analysis - Implications for Porosity-Permeability Transforms
}

\author{
Gregor P. Eberli ${ }^{1}$, Gregor T. Baechle ${ }^{1}$, Ralf Weger ${ }^{1}$, and Jose-Luis. Massaferro ${ }^{2}$ \\ ${ }^{1}$ Comparative Sedimentology Laboratory, \\ University of Miami, \\ 4600 Rickenbacker Cswy, \\ Miami, FL 33149 \\ ${ }^{2}$ Shell International E\&P, \\ Carbonate Development Team, \\ Volmerlaan 8, 2280 AB Rijswijk, \\ The Netherlands
}

\section{Key Findings}

- Five relevant digital image analysis parameters for fluid flow are obtained from 2-D image analysis of carbonate rocks: 2-D image porosity, amount of pores, pore shape, total perimeter per area and dominant pore size range.

- Micro-porosity leads to low permeable, highly porous rocks. 2-D image porosity represents the macro-porosity because the resolution subtracts the small pores from the porosity. Compared to the total porosity, it improves the prediction of permeability by an order of magnitude in the high porosity range.

- Pore shape factor $\gamma$ reduces the uncertainty in permeability prediction to 2 orders of magnitude. Pore shape factor analysis is restricted to samples with more than 4000 pores $/ \mathrm{cm}^{2}$. CT scans of plugs reveals that the pore shape factor $\gamma$ is a relatively constant measure of the pore shape (+/- 0.2) throughout the sample plug.

- The larger the average pore size, the higher the velocity at a given porosity. The average pore size can be quantified by the upper limit of the dominant pore size range.

\section{Introduction}

Carbonate rocks show often a lack of correlation between porosity and other physical properties, in particular permeability and porosity. The high diagenetic potential of carbonates result in intense alteration of the pore structure, which can lead to a decrease of effective porosity for flow and wave propagation. Permeability and elastic properties are strongly related to the rocks pore structure. As a result, samples of equal porosity can exhibit a wide variation of permeability and velocity. Two-dimensional digital image analysis can be used to quantify the pore structure, not requiring any knowledge of pore type or diagenesis of the sample. Although digital image analysis technique is performed on thin sections, physical properties are related to the three dimensional structure of the rock. Nevertheless our study shows that incorporating two-dimensional information from thin sections provides improved estimates for permeability and velocity prediction of the 
strata. The technique has the potential to be applied to very small samples, such as cuttings, and thus improve the characterization of reservoir rocks without complete core recovery.

\section{Method}

We developed a digital image analysis toolbox and designed a workflow that combines high resolution full thin section image acquisition and the use of XPL variation into a streamlined digital image analysis process. Images are acquired at $5 \mu \mathrm{m} / \mathrm{pixel}$ resolution and full thin section photomicrographs are constructed from plain-polarized light and XPL at different angles. A XPL variation attribute is calculated from several images acquired at different angles. This attribute is used as additional information during image segmentation. In particular, the XPL attribute is used to distinguish air bubbles in pore spaces not filled with dyed resign (Fig. 1). In addition, the use of fully integrated relational database facilitates the analysis of both physical and image derived parameters from within a single software application.

The XPL method produces a more accurate segmentation into binary images and allows for more reliable porosity estimates and shape parameters. In addition, the use of open scripting language (Matlab ${ }^{\odot}$ or Scilab ${ }^{\odot}$ ) and the resulting batch capability provides a very efficient method for segmentation of large numbers of images. As a result, statistically more meaningful results can be derived quickly from a group of rock samples. Visual parameter analysis through the use of image- parameter cross plots eliminates the ambiguities introduced by verbal texture and pore type classification.

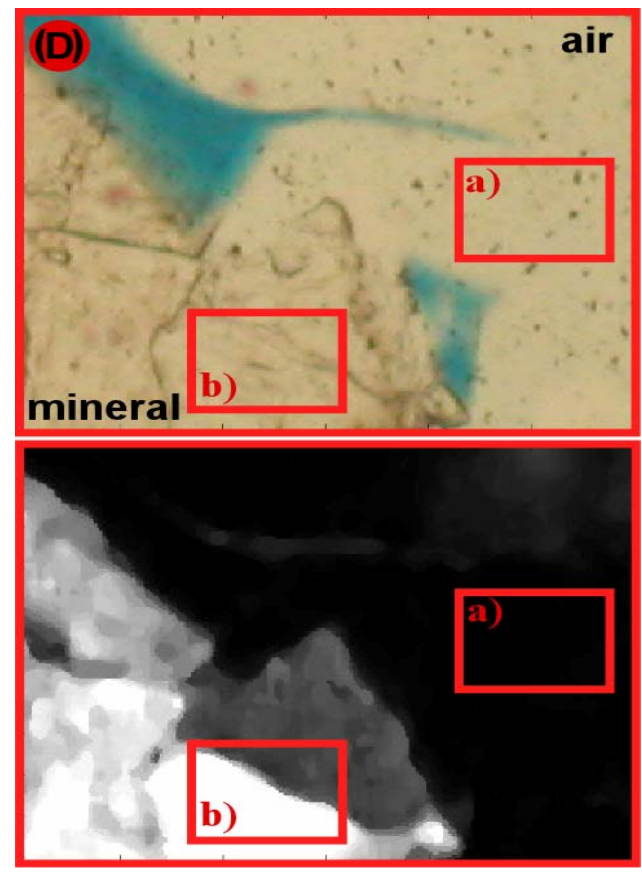

Figure 1. Image (A) acquired using plain polarized light shows a thin section photomicrograph of a carbonate sample from the Bahamas that is impregnated with blue epoxy resin. Minerals and grains are beige while pore space is blue except for an air bubble that is identical in color to the matrix minerals. XPL is capable of destinguishing air bubbles (a) from minerals (b).

Pore shape factor $\gamma$, number of pores, 2-D image porosity, total perimeter and pore sizes at 10,50 and $90 \%$ coverage of cumulative 2-D porosity have been extracted from over 
180 binary images acquired from thin sections of carbonate plug. Velocity, porosity and permeability values have been measured on the corresponding plugs.

\section{Results}

2-D macro-porosity from image analysis:

Macro-porosity is here defined by pores, which are larger than 30 microns, i.e., the thickness of the thin section. In combination with total porosity, it is used as an indicator of "effective" porosity with respect to fluid flow. Large deviation between image analysis macro-porosity and total porosity indicates significant amount of "in-effective" micropores, which generally do not contribute to fluid flow. Those samples tend to plot at the high porosity marginal spectrum. Using image-porosity instead of total porosity reduces the uncertainty in prediction of permeability from 4 to 3 orders of magnitude (Fig.2).
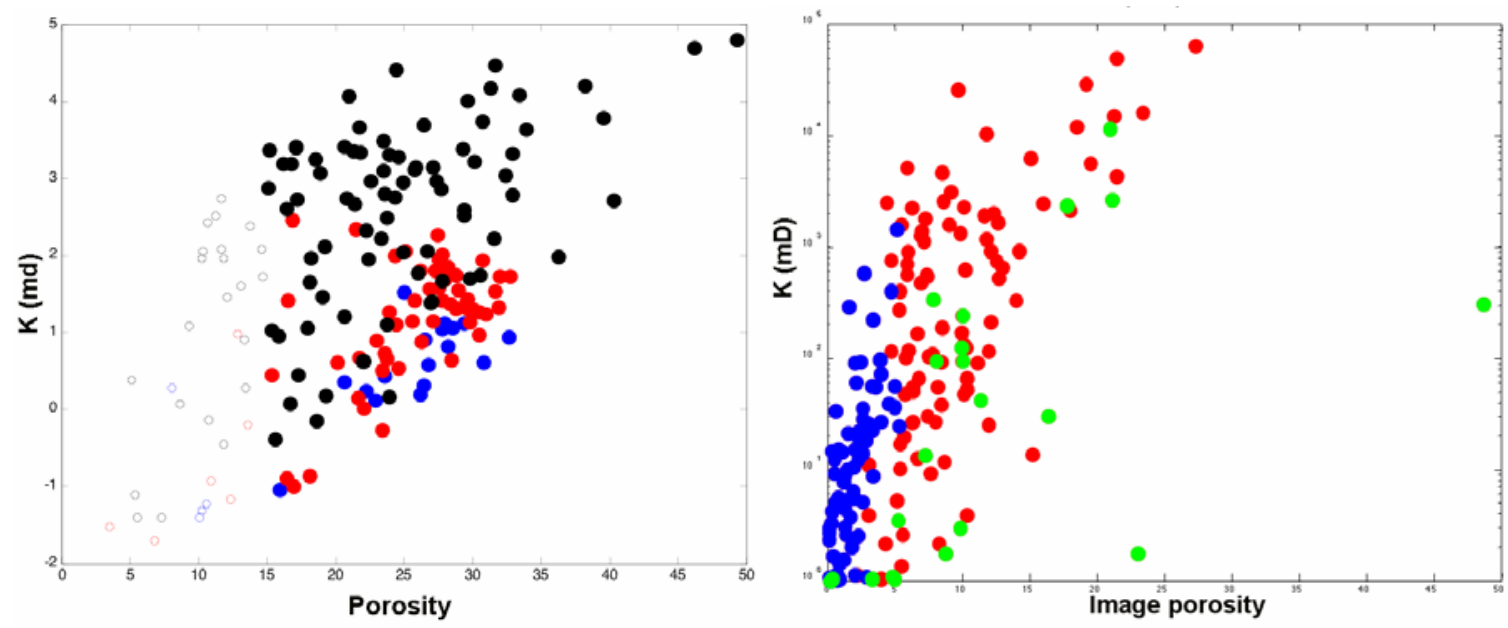

Figure 2: Permeability versus porosity correlation is characterized by large uncertainties. The "image porosity" that calculates porosity from digital image analysis has a better correlation mainly because it eliminates the microporosity.

Pore shape factor:

Anselmetti et al. 1998 established a correlation of permeability and pore shape factor $\gamma$ for carbonate samples (Anselmetti et al. 1998). This value is defined as:

$$
\gamma=\frac{P}{2 \sqrt{\pi A}}
$$

where

$\mathrm{P}=$ Perimeter [mm], $\mathrm{A}=$ Area $\left[\mathrm{mm}^{2}\right]$ and $\gamma=$ pore shape parameter [-].

By using the square root of the area, the pore shape $\gamma$ becomes a dimensionless parameter. For a perfectly round shape, the $\gamma$-value is one and any derivation from this form results in an increase of the $\gamma$-value. The average value of $\gamma$ of one sample is calculated by weighing the individual $\gamma$ by the pore size: 


$$
\bar{\gamma}=\frac{\sum_{i}\left(A_{i} \gamma_{i}\right)}{\sum_{i} A_{i}}
$$

This procedure avoids a high number of insignificant, small pores with specific geometries dominating a few larger pores with a different geometry, which, due to their size, are much more important for the properties of the whole rock. Compared to the porosity-permeability trend, pore shape factor $\gamma$ reduces uncertainty in permeability prediction from 3 to 2 orders of magnitude (Fig. 3).
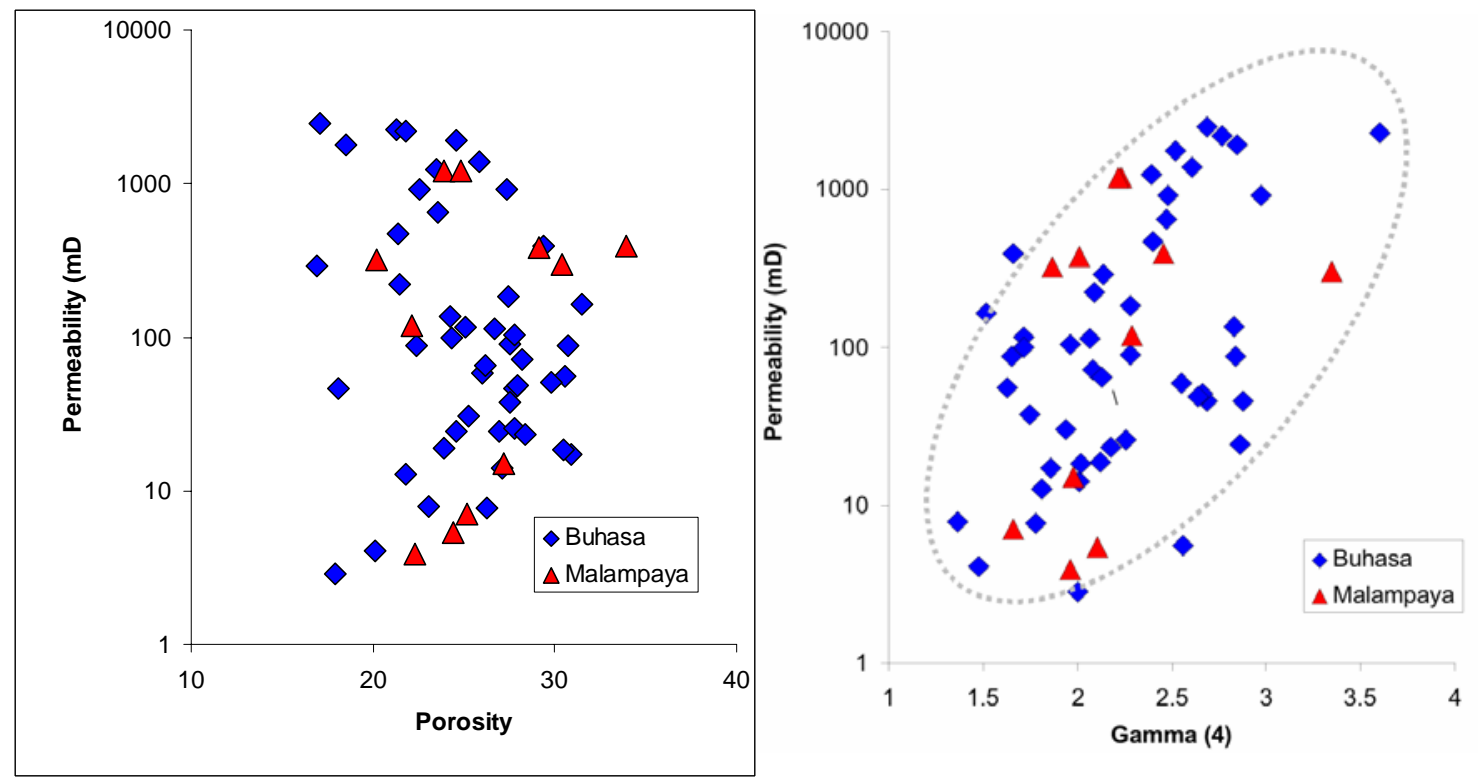

Figure 3: The pore-shape factor $\gamma$ has a better correlation to the permeability than porosity, despite the fact that only the pore shape is taken into account.

Total perimeter:

The measure of total perimeter normalized by the analyzed area can be used to separate "in-effective" porosity from "effective" porosity of the pore network. It is particularly useful in separating high and low permeability samples. Samples with a total perimeter of more than $700 \mathrm{~cm} / \mathrm{cm}^{2}$ have permeabilities below $200 \mathrm{mD}$, and rocks with total perimeter over $1500 \mathrm{~cm} / \mathrm{cm}^{2}$ show no higher permeabilities than $10 \mathrm{mD}$ (Fig. 4). This implies that the more complex and tortuous the pore system with the higher the total perimeter value are less permeable.

Cumulative 2-D porosity:

The dominant pore size is taken as the pore size, at which $50 \%$ of the thin sections 2-D porosity is covered. Within a given porosity range, plugs dominated by small pores tend to have a lower permeability than rocks dominated by large pores. 


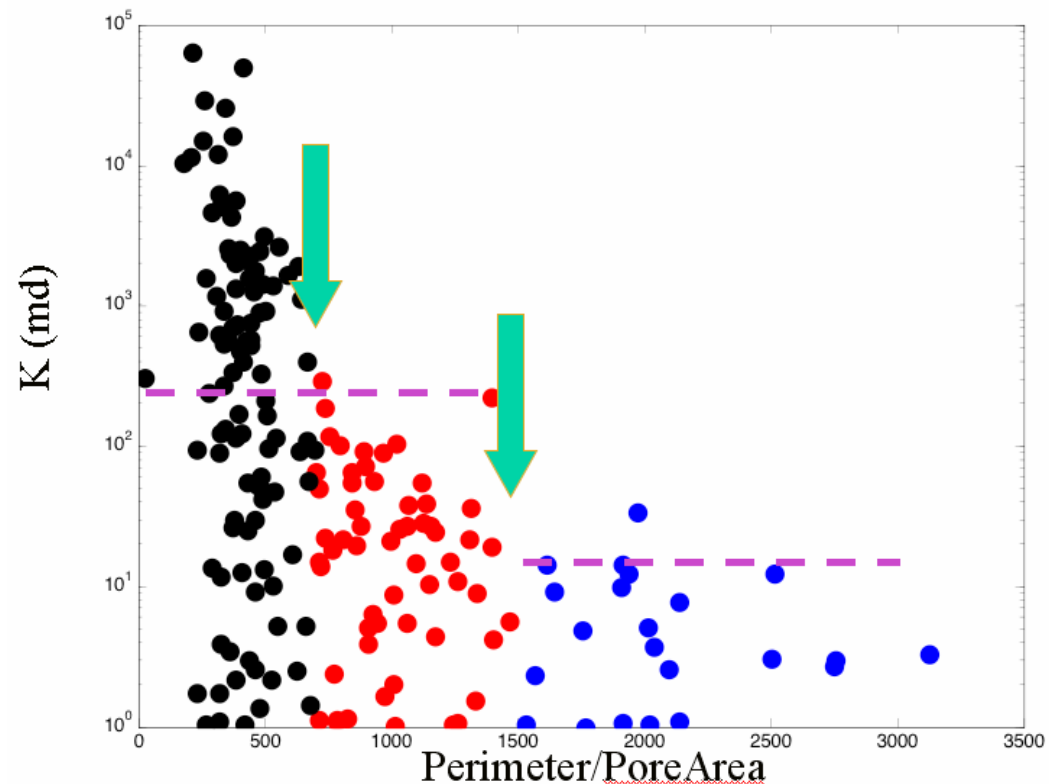

Figure 4: Small numbers of Perimeter/PoreArea values indicate small and tortuous pore systems. High permeability is only found in samples with small values.

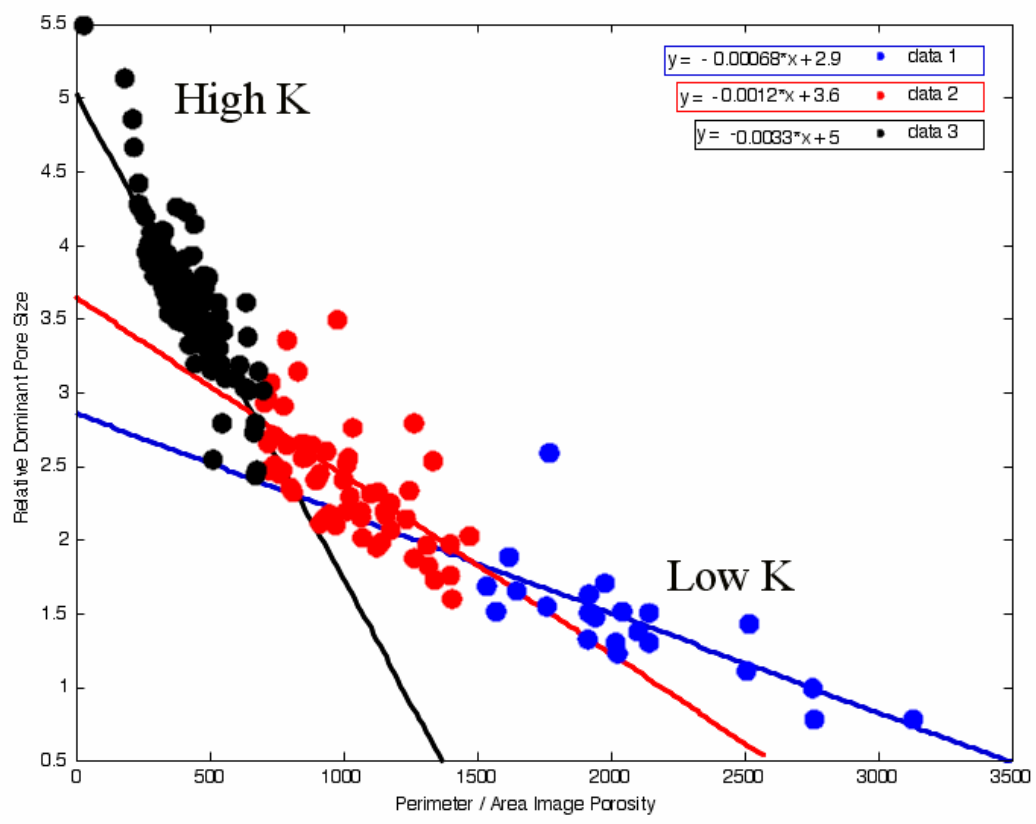

Figure 5: The best correlation is achieved when several digital image analysis parameters are combined, as illustrated in this combination or dominant pore size versus perimeter/pore area.

\section{References:}

Anselmetti, F.S., Lüthi, S. and Eberli, G. (1998). Quantitative characterization of carbonate pore systems by digital image analysis, AAPG Bulletin, Vol. 82, No. 10, p. 1815-1836. 